\title{
Effects of two novel amino acid substitutions on the penicillin binding properties of the PBP5 C-terminal from Enterococcus faecium
}

\author{
CHENGJIANG ZHOU ${ }^{1 *}$, HAIYING NIU ${ }^{2 *}$, HUI YU ${ }^{3}$, LISHE ZHOU ${ }^{1}$ and ZHANLI WANG ${ }^{2,4}$ \\ ${ }^{1}$ Department of Medical and Cell Biology, Baotou Medical College; ${ }^{2}$ Clinical Laboratory, The First Affiliated Hospital, \\ Baotou Medical College; ${ }^{3}$ Clinical Laboratory, The Second Affiliated Hospital, Baotou Medical College; \\ ${ }^{4}$ Department of Epidemiology, School of Public Health, Baotou Medical College, Baotou 014010, P.R. China
}

Received August 20, 2014; Accepted June 5, 2015

DOI: $10.3892 / \mathrm{mmr} .2015 .4057$

\begin{abstract}
The low-affinity penicillin-binding protein (PBP)5 is responsible for resistance to $\beta$-lactam antibiotics in Enterococcus faecium. (E. faecium). In order to evaluate more fully the potential of this species for the development of resistance to $\beta$-lactam antibiotics, the present study aimed to examine the extent of penicillin-binding protein (PBP) variations in a collection of clinical E. faecium isolates. In the present study, the C-terminal domain of PBP5 (PBP5-CD) of 13 penicillin-resistant clinical isolates of $E$. faecium were sequenced and the correlation between penicillin resistance and particular amino acid changes were analyzed. The present study identified for the first time, to the best of our knowledge, two novel substitutions (Tyr460Phe and Ala462Thr or Val462Thr) of E. faecium PBP5-CD. The covalent interaction between penicillin and PBP5-CD was also investigated using homology modeling and molecular docking methods. The theoretical calculation revealed that Phe460 and Thr462 were involved in penicillin binding, suggesting that substitutions at these positions exert effects on the affinity for penicillin, and this increased affinity translates into lower resistance in vitro.
\end{abstract}

\section{Introduction}

Enterococcus faecium has emerged as an important cause of nosocomial infection (1). It is resistant to a variety of antimicrobial agents, including $\beta$-lactam antibiotics. The contribution of penicillin-binding protein 5 (PBP5) to $\beta$-lactam resistance

Correspondence to: Professor Zhanli Wang, Clinical Laboratory, The First Affiliated Hospital, Baotou Medical College, 41 LinYin Road, Baotou 014010, P.R. China

E-mail: wang.zhanli@hotmail.com

*Contributed equally

Key words: penicillin binding protein 5, Enterococcus faecium, penicillin resistance, homology modeling, molecular docking has been intensely investigated $(2,3)$. The existence of the low-affinity PBP5 has been found to be involved in $\beta$-lactam resistance within laboratory mutants and clinical isolates of E. faecium. Previously, it was demonstrated that mutations in amino acids around the active site can alter the affinities of PBP5 for $\beta$-lactams. Sequence data has revealed the presence of amino acid mutations within the C-terminal domain of PBP5 (PBP5-CD) (4). Notably, certain mutations have been revealed to be significantly associated with $\beta$-lactam resistance $(5,6)$.

The crystal structures of PBP5 from Pseudomonas aeruginosa, Escherichia coli and Haemophilus influenzae have been previously determined (7-9). These structures reveal the spatial arrangement of proteins, which explain the reason for the observed substrate specificity of PBP5. However, the crystal structure of PBP5 from E. faecium has not been determined. The present study aimed to examine the PBP5-CD variants of 13 clinical isolates with different levels of penicillin resistance. The impacts of novel amino acid alterations of E. faecium PBP5-CD on penicillin binding were analyzed using homology modeling and molecular docking methods. The results of the present study may assist in investigating the mechanisms involved in $\beta$-lactam resistance in E. faecium. Additionally, the binding patterns obtained in the present study may be useful in the development of novel antibiotics against $\beta$-lactam resistant $E$. faecium.

\section{Materials and methods}

Bacterial strains and identification. A total of 13 clinical isolates of E. faecium were collected from local hospitals in Inner Mongolia, China. Institutional ethical clearance was obtained. The specimens were isolated from various clinical samples, as described previously (10), including urine (9/13), orotracheal fluid (1/13), vaginal/semen swab (1/13), pus (1/13) and blood (1/13). A VITEK 2 Compact system (BioMérieux, Ltd., Marcy l'Etoile, France) and polymerase chain reaction (PCR) analysis were used to identify E. faecium. . In brief, PCR amplification was performed using $1 \mu 1$ template DNA, $1 \mu \mathrm{l}$ of each primer (100 pmol), and $25 \mu \mathrm{l}$ 2X PCR Master Mix (BioSci Biotech, Hangzhou, China) in a total volume of $50 \mu \mathrm{l}$. A C1000 Touch thermocycler (Bio-Rad Laboratories, Inc., 
Hercules, CA, USA) was programmed with an initial denaturation step at $94^{\circ} \mathrm{C}$ for $3 \mathrm{~min} ; 35$ cycles of denaturation at $94^{\circ} \mathrm{C}$ for $30 \mathrm{sec}$, annealing at $54^{\circ} \mathrm{C}$ for $30 \mathrm{sec}$ and elongation at $72^{\circ} \mathrm{C}$ for $1 \mathrm{~min}$, followed by a final extension at $72^{\circ} \mathrm{C}$ for $5 \mathrm{~min}$. The PCR products were analyzed using electrophoresis on a $1 \%$ agarose gel stained with ethidium bromide (Sangon Biotech, Co., Ltd., Shanghai, China). The PCR primers for the ddlE gene were forward 5'-TTGAGGCAGACCAGATTGACG-3' and reverse 5'-TATGACAGCGACTCCGATTCC-3' (11). The present study was approved by the Ethics Comittee of Baotou Medical College, Baotou, Chuna. All subjects provided written informed consent.

Susceptibility assessment. The antimicrobial susceptibility of the of E. faecium isolates were determined using a disk diffusion technique with the Kirby-Bauer (K-B) test method, according to the standards and interpretive criteria described by the Clinical and Laboratory Standards Institute (CLSI) (12). The drugs used for disc diffusion assessment were obtained from Oxoid, Ltd. (Basingstoke, UK) in the following concentrations: Chloramphenicol $(30 \mu \mathrm{g})$, gentamicin $(120 \mu \mathrm{g})$, norfloxacin $(30 \mu \mathrm{g})$, ciprofloxacin $(5 \mu \mathrm{g})$, ampicillin $(10 \mu \mathrm{g})$, tetracycline $(30 \mu \mathrm{g})$, penicillin $(10 \mathrm{IU})$, erythromycin $(15 \mu \mathrm{g})$ and streptomycin $(300 \mu \mathrm{g})$. E. faecalis ATCC 29212 (susceptible) and E.fecalis ATCC 51299 (resistant) strains were used as the quality control strains for the determination of antimicrobial susceptibility (12).

Western blotting. Western blotting was performed to detect the expression levels of PBP5 in the 13 clinical E.faecium isolates, as described previously (13). Briefly, proteins were prepared from $3 \mathrm{ml}$ cultures of the E. faecium sample in the stationary growth phase. For western blot analysis, $15 \mu \mathrm{l}$ of the proteins were separated on SDS polyacrylamide gel and transferred onto polyvinylidene fluoride (PVDF) membranes (Sangon Biotech, Co., Ltd.), blocked for $1 \mathrm{~h}$ at $37^{\circ} \mathrm{C}$ in PBS containing non-fat dry milk (Sangon Biotech, Co., Ltd.) and incubated overnight at $4^{\circ} \mathrm{C}$ with the primary antibody (polyclonal anti-PBP5; Vital River Laboratories, Beijing, China) diluted 1:1,000 in PBS. Following washing in Tween $200.05 \%$ v/v in Tris-buffered saline, the PVDF membranes were incubated for $1 \mathrm{~h}$ at room temperature with peroxidase-conjugated goat anti-rabbit secondary antibody (cat. no. C006387; Sangon Biotech, Co., Ltd.) diluted 1:2,000 with PBS. Signals were detected using enhanced chemiluminescence substrate (Sangon Biotech, Co., Ltd.). Preparation of the anti-PBP5 antibody was performed, as described previously (14).

$P C R$ and DNA sequencing. The total DNA template was extracted from E. faecium using a Bacteria Genomic DNA Extraction kit (BioSci Biotech, Hangzhou, China), according to the manufacturer's instructions. PCR was used to detect a 794 bp fragment from E. faecium encoding PBP5 CD. The PCR conditions were as follows: Initial denaturation at $94^{\circ} \mathrm{C}$ for $3 \mathrm{~min} ; 40$ cycles of denaturation at $94^{\circ} \mathrm{C}$ for $30 \mathrm{sec}$, annealing at $52^{\circ} \mathrm{C}$ for $1 \mathrm{~min}$ and elongation at $72^{\circ} \mathrm{C}$ for $2 \mathrm{~min}$, followed by a final extension at $72^{\circ} \mathrm{C}$ for $5 \mathrm{~min}$. The PCR products were analyzed using electrophoresis on agarose gel, as described above. The sequence of the PCR primers were as follows: forward 5'-CGGGATCTCACAAGAAGAT-3' and reverse 5'-TTATTGATAATTTTGGTT-3'. The PCR products were purified using the DNA Agarose Gel Extraction Kit (Sangon Biotech, Co., Ltd.) and were sequenced by Sangon Biotech Co., Ltd. using a Primer-Walking method.

Homology modeling. The homology model of PBP5-CD from E. faecium was constructed using the Modeller program (version 9.10) and the structure of PBP2a from the $27 \mathrm{r}$ methicillin-resistant Staphylococcus aureus strain (University of British Columbia, British Columbia, Canada) was used as a template (PDB code, 1VQQ). The model structure was subsequently refined by energy minimization in the presence of explicit solvent model TIP3P water using the CHARMm program (version c29b2). The obtained structure was assessed using the Profiles-3D program within Insight II 2005 (Accelrys, San Diego, CA, USA).

Molecular docking. The Affinity program within Insight II 2005 (Accelrys) was used for docking experiments, as described previously (15). The consistent valence force field was selected prior to performing docking calculations. Since the active site of PBP5-CD is conserved, the crystal structure of covalent complexes of a $\beta$-lactam antibiotic with $E$. coli PBP5 (PDB code, 3MZD) was used as a reference structure, to identify the initial binding site of penicillin in the E. faecium PBP5-CD.

\section{Results and Discussion}

Identification and susceptibility of E. faecium. All 13 isolates were identified as E. faecium using the VITEK 2 Compact system and PCR assay. The K-B method revealed resistance to penicillin, with a zone of $32 \mathrm{~mm}$ for strain 7 and $\geq 64 \mathrm{~mm}$ for all other isolates. In the present study, the resistance breakpoint was $\geq 16 \mathrm{~mm}$, according to CLSI guidelines (12). These results provided clear evidence that strain 7 exhibited a lower level of penicillin resistance, compared with the other isolates. Considering the crucial role of PBP5 in $\beta$-lactam resistance, it was suggested that the different levels of penicillin resistance between these E. faecium isolates may have been due to the presence of low-affinity PBP5 or the production of PBP5.

Expression of E. faecium pbp5 genes. In order to identify and understand the mechanisms underlying penicillin resistance among the clinical E.faecium isolates, the expression levels of pbp5 genes were determined using western blotting (data was not shown). The results demonstrated that the expression levels of the pbp5 genes were similar, indicating that the production of PBP5 did not account for the different levels of penicillin resistance, which were observed in the E. faecium isolates.

Alternations in the E. faecium PBP5-CD. The PBP5-CD variants from the isolates were further investigated in the present study. The PBP5-CD genes of the 13 E. faecium isolates were amplified and sequenced. From the primary structure alignment, a consensus sequence was observed (Fig. 1A). As shown in Fig. 1B, an evolutionary path, as depicted by the evolutionary tree, was observed. Unlike the PBP5 in the majority of isolates, which was identified with the zone of $\geq 64 \mathrm{~mm}$, the PBP5 variant of strain 7, with the zone of $32 \mathrm{~mm}$, had two 
A

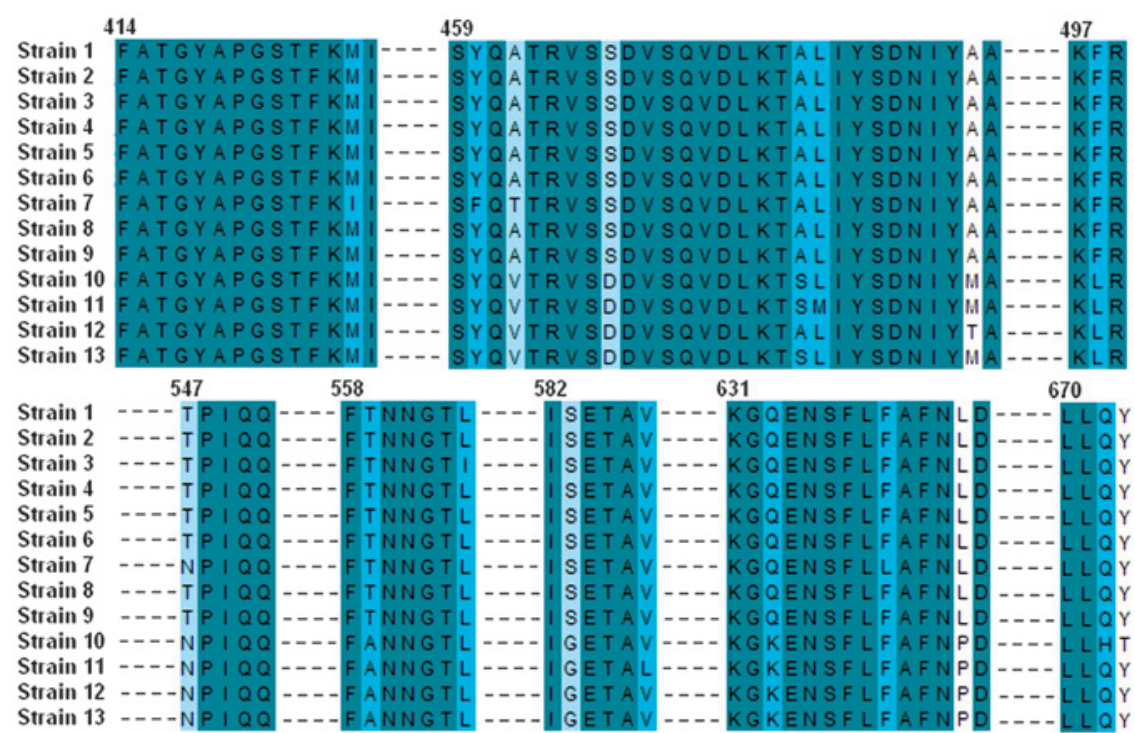

B

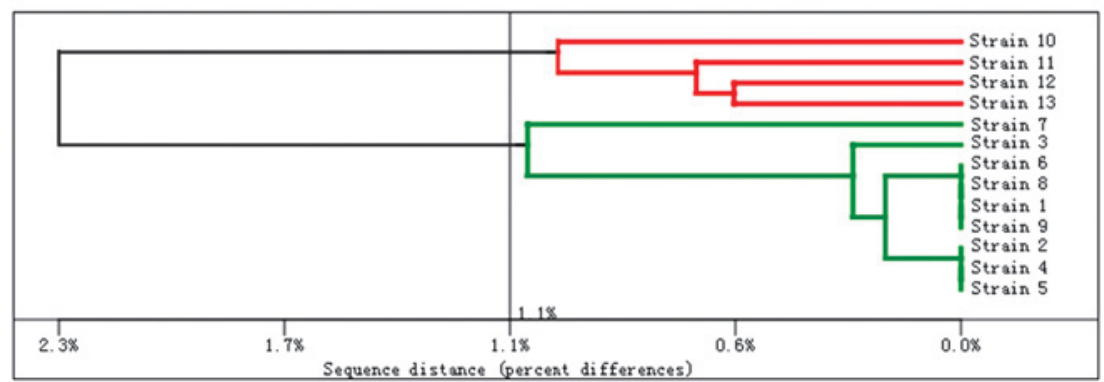

Figure 1. (A) Sequence alignments and (B) evolutionary tree of the C-terminal domains of PBP5 from E. faecium clinical isolates.

A

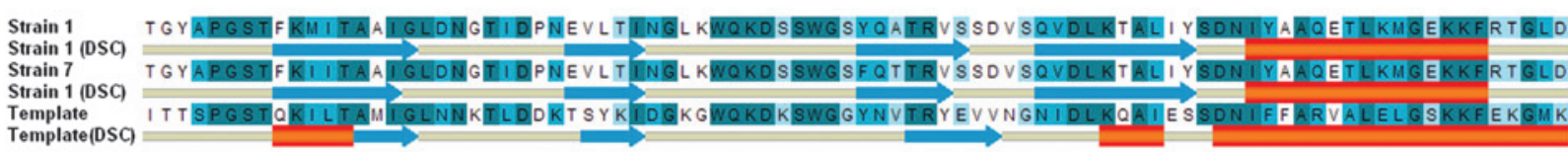

Strain 1 KFI FGEDLDLPISMNPAQISNEDSFNSDILLADTGYGQGELLITPI QQAAMYSVFTNNGTLVYPKLIADKETKD-KKNVISETAVQT II Strain 1 (DSC) Strain 1 (DSC)
Template Template(DSC)

Strain 1 VPDLREVVQOVNGTAHSLSALGIPLAAKTGTAEI KEKQDVKGQENSFLFAFNLDNQGYMMVSMLENKEDDDSATKRA- - SELLQYLN

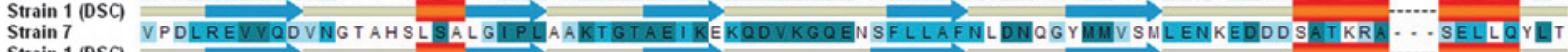
Strain 1 (DSC)
Template
Template(DSC)

B

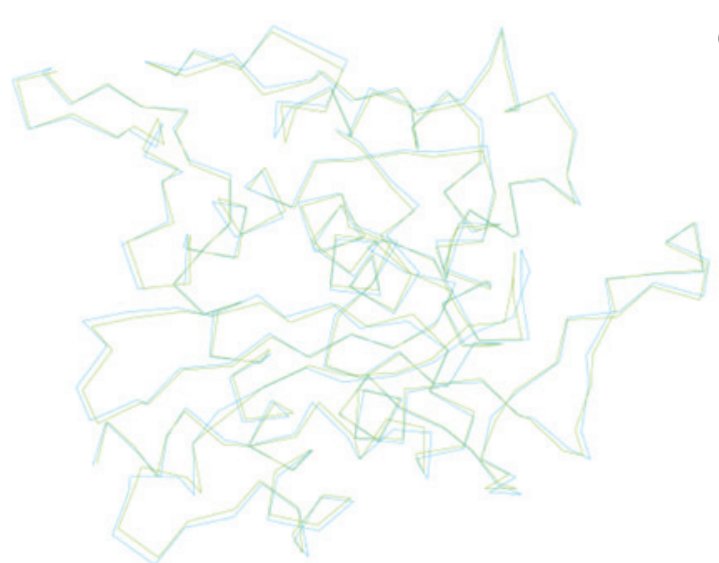

C
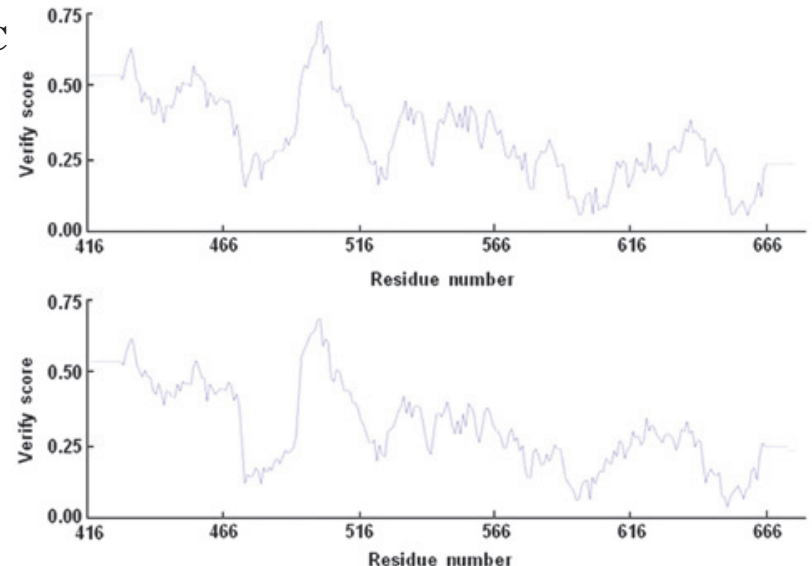

Figure 2. Homology modeling of PBP5-CD. (A) Sequence alignments between the PBP5-CDs and the template. (B) Homology models of the strain 1 (green) and strain 7 (blue) PBP5-CDs. (C) Profiles-3D results of the strain 1 (upper) and strain 7 (lower) PBP5-CD models. Residues with positive compatibility scores were reasonably folded. PBP5-CD, C-terminal domain of PBP5; DSC, differential scanning calorimetry. 
A
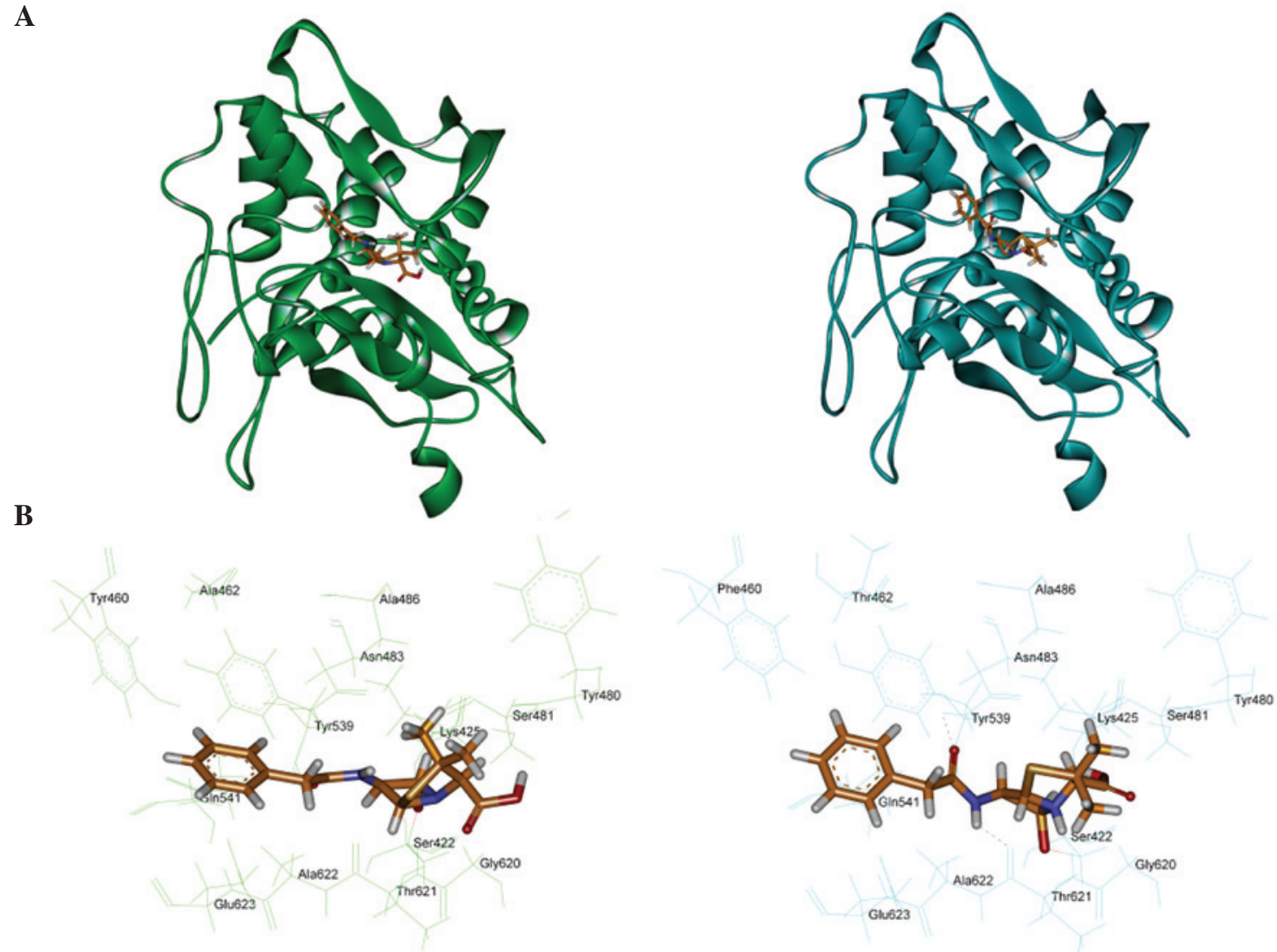

Figure 3. Predicted models of PBP5-CD complexed with penicillin. (A) Docking orientation of penicillin into the strain 1 (green) and strain 7 (blue) PBP5-CD models. (B) Binding of penicillin to the active site in the strain 1 (green) and strain 7 (blue) PBP5-CD models. PBP5-CD, C-terminal domain of PBP5.

novel changes at positions 460 and 462 . This is the first report, to the best of our knowledge, to describe the Tyr460Phe and Ala462Thr or Val462Thr PBP5-CD mutations in E. faecium isolates. These substitutions are located in a loop, which forms the outer edge of the active site, and are in close proximity to the inserted serine at position 466 . Therefore, the present study hypothesized that these mutations may be involved in the binding of penicillin to PBP5-CD, consequently resulting in a lower level of resistance towards penicillin.

Homology model of E. faecium PBP5-CD. To further confirm the above hypothesis, homology models of PBP5-CD were constructed. Strain 1 and strain 7, which exhibited differences in the resistance to penicillin, were included in this experiment. According to the sequence alignment result, the percentages of identity of strain 1 and strain 7 with the template were 36.7 and $37.1 \%$, respectively. Sequence alignment analysis also revealed the predicted secondary structures of PBP5-CD, which indicated that these proteins were structurally similar to one another (Fig. 2A). The homology models of the PBP5-CD of strain 1 and strain 7 were subsequently constructed, based on the structure of the template (Fig. 2B), and the quality of the modeled structures was determined. All residues were in sterically allowed regions of the Ramachandran plot. In addition, the overall self-compatibility scores for the predicted structures were all above the expected values (Fig. 2C). These results indicated that the predicted models were reliable. Subsequently, the two predicted structures of PBP5-CD were compared, to understand the effects of the substitutions on the spatial arrangement. The superposition of $\mathrm{C} \alpha$ atoms from the PBP5-CD of strain 1 onto the corresponding atoms of strain 7 provided a root mean square deviation of $0.529 \AA$ (Fig. 2B), which indicated that certain substitutions were able to produce minor conformational changes, which may have the effect of altering the orientation of residues in the binding sites.

Detection of binding between penicillin and PBP5-CD. In order to obtain additional evidence to support the hypothesis that Phe 460 and Thr462 are involved in penicillin binding, molecular docking was performed. However, calculation of covalent bonds is challenging due to the principle limitation of molecular simulation methods, particularly for PBP5, and no docking methods have been previously reported. In order to set up a reliable theoretical method to fully understand the binding mode between PBP5-CD and penicillin, the active site of PBP5-CD was obtained by using the crystal structure of E. coli $\mathrm{PBP} 5$ as the template. Fig. 3 shows the covalent binding mode of penicillin with PBP5-CD. The penicillin covalently interacted with the serine at position 422 of the active-site, which was consistent with the results of a previous study (4). For the PBP5-CD of strain 7, the results demonstrated that hydrophobic groups were positioned in lipophilic pockets, which were created by Tyr539, Phe460 and Thr 462. The theoretical calculation also indicated that penicillin formed two hydrogen bonds with Asn483 and Thr621. However, no hydrogen bonding was observed in strain 1 , and the 
replacement of Phe460 and Thr462 with Tyr460 and Ala462 resulted in a reduction in hydrophobic interactions.

In conclusion, Phe460 and Thr462 in strain 7 may increase the ability of PBP5 to bind to penicillin and affect the level of penicillin resistance of E. faecium. This is the first report, to the best of our knowledge, demonstrating that the two residues, Phe460 and Thr462, were responsible for the lower penicillin resistance of E. faecium observed in vitro.

\section{Acknowledgements}

The present study was supported by the Natural Science Foundation of Inner Mongolia Autonomous Region of China (grant. nos. 2014JQ04 and 2012MS1159) and the National Natural Science Foundation of China (grant. no. 81460049).

\section{References}

1. Murray BE: The life and times of the Enterococcus. Clin Microbiol Rev 3: 46-65, 1990.

2. Rice LB, Carias LL, Hutton-Thomas R, Sifaoui F, Gutmann L and Rudin SD: Penicillin-binding protein 5 and expression of ampicillin resistance in Enterococcus faecium. Antimicrob Agents Chemother 45: 1480-1486, 2001.

3. Rybkine T, Mainardi JL, Sougakoff W, Collatz E and Gutmann L: Penicillin-binding protein 5 sequence alterations in clinical isolates of Enterococcus faecium with different levels of $\beta$-lactam resistance. J Infect Dis 178: 159-163, 1998.

4. Rice LB, Bellais S, Carias LL, Hutton-Thomas R, Bonomo RA, Caspers P, Page MG and Gutmann L: Impact of specific pbp5 mutations on expression of beta-lactam resistance in Enterococcus faecium. Antimicrob Agents Chemother 48 3028-3032, 2004.

5. Hsieh SE, Hsu LL, Hsu WH, Chen CY, Chen HJ and Liao CT: Importance of amino acid alterations and expression of penicillin-binding protein 5 to ampicillin resistance of Enterococcus faecium in Taiwan. Int J Antimicrob Agents 28: 514-519, 2006.
6. Poeta P, Costa D, Igrejas G, Sáenz Y, Zarazaga M, Rodrigues J and Torres C: Polymorphisms of the pbp5 gene and correlation with ampicillin resistance in Enterococcus faecium isolates of animal origin. J Med Microbiol 56: 236-240, 2007.

7. Smith JD, Kumarasiri M, Zhang W, Hesek D, Lee M, Toth M, Vakulenko S, Fisher JF, Mobashery S and Chen Y: Structural analysis of the role of Pseudomonas aeruginosa penicillin-binding protein 5 in $\beta$-lactam resistance. Antimicrob Agents Chemother 57: 3137-3146, 2013.

8. Nicola G, Tomberg J, Pratt RF, Nicholas RA and Davies C: Crystal structures of covalent complexes of $\beta$-lactam antibiotics with Escherichia coli penicillin-binding protein 5: Toward an understanding of antibiotic specificity. Biochemistry 49: 8094-8104, 2010.

9. Kawai F, Clarke TB, Roper DI, Han GJ, Hwang KY, Unzai S, Obayashi E, Park SY and Tame JR: Crystal structures of penicillin-binding proteins 4 and 5 from Haemophilus influenzae. J Mol Biol 396: 634-645, 2010.

10. Li W, Li J, Wei Q, Hu Q, Lin X, Chen M, Ye R and Lv H: Characterization of aminoglycoside resistance and virulence genes among Enterococcus spp. isolated from a hospital in China. Int J Environ Res Public Health 12: 3014-3025, 2015.

11. Macovei L and Zurek L: Ecology of antibiotic resistance genes: characterization of enterococci from houseflies collected in food settings. Appl Environ Microbiol 72: 4028-4035, 2006.

12. Clinical and Laboratory Standards Institute: Performance Standards for Antimicrobial Susceptibility Testing: Twentieth Informational Supplement. Wayne, PA: Clinical and Laboratory Standards Institute, p. M100-S23, 2013.

13. Rybkine T, Mainardi JL, Sougakoff W, Collatz E and Gutmann L: Penicillin-binding protein 5 sequence alterations in clinical isolates of Enterococcus faecium with different levels of beta-lactam resistance. J Infect Dis 178: 159-163, 1998.

14. Hsieh SE, Hsu LL, Hsu WH, Chen CY, Chen HJ and Liao CT: Importance of amino acid alterations and expression of penicillin-binding protein 5 to ampicillin resistance of Enterococcus faecium in Taiwan. Int J Antimicrob Agents 28: 514-519, 2006

15. Lei M, Zhao X, Wang Z and Zhu Y: Pharmacophore modeling, docking studies and synthesis of novel dipeptide proteasome inhibitors containing boron atoms, J Chem Inf Model 49: 2092-2100, 2009. 